\title{
Altitude e coordenadas geográficas na estimativa da temperatura mínima média decendial do ar no Estado do Rio Grande do Sul
}

\author{
Alberto Cargnelutti Filho( ${ }^{(1)}$, Jaime Ricardo Tavares Maluf(2), Ronaldo Matzenauer ${ }^{(3)}$ e Álvaro Prestes Stolz ${ }^{(4)}$
}

\begin{abstract}
(1)Universidade Estadual Paulista, Fac. de Ciências Agrárias e Veterinárias, Dep. de Ciências Exatas, Via de Acesso Prof. Paulo Donato Castellane, s/no-, CEP 14884-900 Jaboticabal, SP. E-mail: cargnelutti@fcav.unesp.br (2)Embrapa Trigo, Rod. BR 285, Km 174, CEP $99001-970$ Passo Fundo, RS. E-mail: maluf@cnpt.embrapa.br (3)Fundação Estadual de Pesquisa Agropecuária, Rua Gonçalves Dias, no 570, Menino Deus, CEP 90130-060 Porto Alegre, RS. E-mail: ronaldo-matzenauer@fepagro.rs.gov.br (4)Universidade Federal do Rio Grande do Sul, Av. Bento Gonçalves, № 7.712, Agronomia, CEP 91501-970 Porto Alegre, RS. E-mail: stolz@cpovo.net
\end{abstract}

Resumo - O objetivo deste trabalho foi verificar, com dados de temperatura mínima média decendial do ar (Tm) de 41 municípios do Estado do Rio Grande do Sul, de 1945 a 1974, se a Tm pode ser estimada em função da altitude, latitude e longitude. Para cada um dos 36 decêndios do ano, realizaram-se análise de correlação, análise de trilha das variáveis causais - altitude, latitude e longitude - sobre o efeito Tm, e estimaram-se os parâmetros do modelo das equações de regressão linear múltipla, pelo método passo a passo, com teste para saída de variáveis, considerando Tm como variável dependente e altitude, latitude e longitude como variáveis independentes. Na validação dos modelos de estimativa da Tm, usou-se o coeficiente de correlação linear de Pearson, entre a Tm estimada e a Tm observada em dez municípios do Estado, com dados da série de observações meteorológicas de 1975 a 2004. A temperatura mínima média decendial do ar pode ser estimada pelas coordenadas geográficas em qualquer local e decêndio, no Estado do Rio Grande do Sul. A altitude e latitude explicam melhor a variação da Tm.

Termos para indexação: modelagem, clima, temperatura do ar.

\section{Altitude and geographic coordinates in the ten-day mean minimum air temperature estimation in the State of Rio Grande do Sul, Brazil}

\begin{abstract}
The objective of this work was to estimate ten-day mean minimum air temperature (Tm) using altitude and the geographic coordinates latitude and longitude for the Rio Grande do Sul State, Brazil. Normal ten-day mean minimum air temperature of 41 counties in the State of Rio Grande do Sul, from 1945 to 1974 were used. Correlation analysis, path analysis of causal variables (altitude, latitude and longitude) on $\mathrm{Tm}$, and parameters estimate of multiple linear regression equations were performed by the stepwise backward method, using Tm as dependent variable and altitude, latitude and longitude as independent variables, for the 36 ten-day periods of the year. Pearson's linear correlation coefficient between estimated and observed Tm, calculated for tem counties using data of the series of meteorological observations from 1975 to 2004 were used as independent data sets. The ten-day mean minimum air temperature may be estimated from the geographic coordinates in the State of Rio Grande do Sul. Altitude and latitude best explain the variation of the Tm.
\end{abstract}

Index terms: modeling, climate, air temperature.

\section{Introdução}

No estudo da adaptabilidade e comportamento das culturas agrícolas é importante o conhecimento e a análise dos elementos meteorológicos. A relação clima-planta é caracterizada e mensurada por meio da magnitude dessas variáveis e da resposta da planta ao ambiente. Assim, para trabalhos de zoneamento de culturas, indicação de épocas de semeadura, irrigação, determinação de potencial de rendimento, zoneamento de riscos climáticos, crédito e seguro agrícola, usam-se dados meteorológicos horários, diários, decendiais e mensais, conforme o alcance e detalhamento do estudo e do tipo de cultura em questão.

O estudo da disponibilidade térmica do ambiente às culturas agrícolas é realizado com a temperatura em suas diversas modalidades - médias, máximas e mínimas -, medida em estações meteorológicas que, entretanto, nem sempre são em número suficiente e com distribuição geográfica satisfatória. Vários trabalhos têm buscado 
métodos de estimativa de parâmetros meteorológicos, principalmente temperatura, em locais ou regiões carentes de medição.

Para o Estado de São Paulo, Pinto et al. (1972) estabeleceram equações de estimativa das temperaturas médias mensais e anuais, em função da altitude e latitude, alcançando elevados coeficientes de correlação, o que evidenciou boa precisão nas estimativas. Posteriormente, Pinto \& Alfonsi (1974) estimaram temperaturas médias, máximas e mínimas mensais no Estado do Paraná, em função da altitude e latitude, obtendo coeficientes de determinação elevados, mas ressalvam que as estimativas não são válidas para a faixa litorânea do Paraná, em virtude da influência do Oceano Atlântico, e que, nos meses de inverno, ocorreram maiores discordâncias entre os dados calculados e medidos, com coeficientes de determinação de 66 a 85\%.

Alfonsi et al. (1974) realizaram estimaram as normais de temperatura média mensal e anual do Estado de Goiás em função da altitude e latitude e encontraram elevados coeficientes de determinação, mostrando que a variação da temperatura média do ar nesse Estado ocorre, principalmente, em virtude da variação da altitude e da latitude. Encontraram um gradiente de $0,5^{\circ} \mathrm{C}$ a cada $100 \mathrm{~m}$ de deslocamento vertical, e um gradiente de $0,1^{\circ} \mathrm{C}$ para variação de cada grau de latitude, com um valor maior, de $0,6^{\circ} \mathrm{C}$, no inverno.

Recentemente, no Estado de São Paulo, Pedro Júnior et al. (1991) estimaram as temperaturas médias das máximas e mínimas mensais em função da altitude e latitude, e verificaram que as equações de estimativa não são aplicáveis às áreas litorâneas e, no caso de São Paulo, ao Vale do Ribeira de Iguape.

Quanto aos Estados do Rio Grande do Sul (Ferreira et al., 1971) e de Santa Catarina (Ferreira et al., 1974), trabalhos de estimativa das temperaturas médias mensais e anuais do ar, em função das coordenadas geográficas altitude e latitude, revelaram resultados com elevados coeficientes de correlação, evidenciando bom ajuste dos modelos.

Elevados coeficientes de correlação, ao realizar a estimativa da média das temperaturas máximas mensais e anuais do ar em função da altitude e latitude, no Estado do Rio Grande do Sul, foram obtidos por Buriol et al. (1973) e, no Estado de Santa Catarina, por Buriol et al. (1974). Tais equações podem ser usadas para estimar as temperaturas médias das máximas do ar em todo o
Estado, com exceção da faixa litorânea, mencionando que esse efeito de baixa correlação na faixa litorânea é atribuído, provavelmente, à influência das grandes massas de água.

Estefanel et al. (1973) realizaram, no Estado do Rio Grande do Sul, estimativa das médias das temperaturas mínimas mensais e anuais em função da altitude e latitude. Da mesma maneira que Buriol et al. (1973), concluíram que as equações podem ser usadas para estimar as temperaturas médias das máximas, em todo o Estado, com exceção da faixa litorânea. O efeito de baixa correlação na faixa litorânea é atribuído, provavelmente, à influência das grandes lagoas e do oceano.

A estimativa da temperatura do ar mínima, média e máxima mensal e anual, a partir das coordenadas geográficas, tem sido estudada nos estados de Minas Gerais (Coelho et al., 1973; Sediyama \& Melo Júnior, 1998), Espírito Santo (Feitoza et al., 1980a, 1980b) e Piaú (Lima \& Ribeiro, 1998), e em regiões do Brasil (Oliveira Neto et al., 2002; Medeiros et al., 2005).

A ausência de dados meteorológicos referentes a algumas regiões, associada à facilidade de obtenção de coordenadas geográficas e a importância da temperatura em atividades da agricultura justifica a investigação da influência das coordenadas geográficas sobre a Tm e a elaboração de modelos para sua estimação, a partir das coordenadas geográficas. Além disso, estimativas em escala decendial possibilitam estudos mais detalhados em relação às escalas mensal e anual.

Em estudos que exijam o conhecimento das temperaturas, nos âmbitos mesoclimático e microclimático (local), nem sempre existem estações meteorológicas próximas o suficiente para permitir determinar: o valor médio da temperatura mínima do ar, de modo a possibilitar o estabelecimento e o desenvolvimento economicamente adequado de uma cultura, naquele ambiente; ou ainda, o valor da temperatura mínima para início de semeadura em determinado local ou microrregião. O aspecto da análise da temperatura mínima decendial para culturas perenes adquire maior dimensão, pois a adaptabilidade e o desenvolvimento dessa cultura é medida em anos.

O objetivo deste trabalho foi verificar, com dados de 41 municípios do Estado do Rio Grande do Sul, se a temperatura mínima média decendial pode ser estimada em função da altitude, latitude e longitude. 


\section{Material e Métodos}

Foram usados dados de temperatura mínima média decendial do ar (Tm) de 41 estações meteorológicas pertencentes ao 8o Distrito de Meteorologia do Instituto Nacional de Meteorologia, localizadas no Estado do Rio Grande do Sul. As informações foram obtidas em Instituto de Pesquisas Agronômicas (1989), e são referentes ao período com disponibilidade de dados - 1945 a 1974 (Tabela 1).

Tabela 1. Altitude (m) e coordenadas geográficas das estações meteorológicas, localizadas em municípios do Estado do Rio Grande do Sul.

\begin{tabular}{|c|c|c|c|}
\hline \multirow{2}{*}{$\begin{array}{l}\text { Município } \\
\text { Alegrete }\end{array}$} & \multirow{2}{*}{$\begin{array}{c}\text { Altitude } \\
121,00\end{array}$} & \multicolumn{2}{|c|}{$\begin{array}{l}\text { Coordenada geográfica }{ }^{(1)} \\
\text { Latitude }(\mathrm{S}) \text { Longitude }(\mathrm{W})\end{array}$} \\
\hline & & $29^{\circ} 41^{\prime} 47^{\prime \prime}$ & $55^{\circ} 31^{\prime} 15^{\prime \prime}$ \\
\hline Bagé & 242,31 & $31^{\circ} 20^{\prime} 13^{\prime \prime}$ & $54^{\circ} 06^{\prime} 21^{\prime \prime}$ \\
\hline Bento Gonçalves & 640,00 & $29^{\circ} 09^{\prime} 00^{\prime \prime}$ & $51^{\circ} 31^{\prime} 00^{\prime \prime}$ \\
\hline Bom Jesus & 1047,50 & $28^{\circ} 40^{\prime} 10^{\prime \prime}$ & $50^{\circ} 26^{\prime} 25^{\prime \prime}$ \\
\hline Caçapava do Sul & 450,00 & $30^{\circ} 30^{\prime} 32^{\prime \prime}$ & $53^{\circ} 29^{\prime} 22^{\prime \prime}$ \\
\hline Cachoeira do Sul & 72,71 & $30^{\circ} 02^{\prime} 45^{\prime \prime}$ & $52^{\circ} 53^{\prime} 39^{\prime \prime}$ \\
\hline Caxias do Sul & 759,60 & $29^{\circ} 10^{\prime} 25^{\prime \prime}$ & $51^{\circ} 12^{\prime} 21^{\prime \prime}$ \\
\hline Cruz Alta & 472,50 & $28^{\circ} 38^{\prime} 21^{\prime \prime}$ & $53^{\circ} 36^{\prime} 34^{\prime \prime}$ \\
\hline Dom Pedrito & 140,00 & $30^{\circ} 58^{\prime} 57^{\prime \prime}$ & $54^{\circ} 39^{\prime} 56^{\prime \prime}$ \\
\hline Guaporé & 471,51 & $28^{\circ} 55^{\prime} 44^{\prime \prime}$ & $51^{\circ} 54^{\prime} 45^{\prime \prime}$ \\
\hline Irai & 247,10 & $27^{\circ} 11^{\prime} 45^{\prime \prime}$ & $53^{\circ} 14^{\prime} 01^{\prime \prime}$ \\
\hline Itaqui & 76,00 & $29^{\circ} 07^{\prime} 10^{\prime \prime}$ & $56^{\circ} 32^{\prime} 52^{\prime \prime}$ \\
\hline Jaguarão & 50,00 & $32^{\circ} 33^{\prime} 32^{\prime \prime}$ & $53^{\circ} 23^{\prime} 20^{\prime \prime}$ \\
\hline Lagoa Vermelha & 840,00 & $28^{\circ} 13^{\prime} 35^{\prime \prime}$ & $51^{\circ} 30^{\prime} 51^{\prime \prime}$ \\
\hline Marcelino Ramos & 414,17 & $27^{\circ} 27^{\prime} 40^{\prime \prime}$ & $51^{\circ} 54^{\prime} 22^{\prime \prime}$ \\
\hline Mostardas & 3,00 & $31^{\circ} 06^{\prime} 25^{\prime \prime}$ & $50^{\circ} 55^{\prime} 16^{\prime \prime}$ \\
\hline Palmeira das Missões & 634,00 & $27^{\circ} 53^{\prime} 55^{\prime \prime}$ & $53^{\circ} 26^{\prime} 45^{\prime \prime}$ \\
\hline Passo Fundo & 684,05 & $28^{\circ} 13^{\prime} 39^{\prime \prime}$ & $52^{\circ} 24^{\prime} 33^{\prime \prime}$ \\
\hline Pelotas & 13,00 & $31^{\circ} 47^{\prime} 00^{\prime \prime}$ & $52^{\circ} 25^{\prime} 00^{\prime \prime}$ \\
\hline Piratini & 345,00 & $31^{\circ} 26^{\prime} 54^{\prime \prime}$ & $53^{\circ} 06^{\prime} 09^{\prime \prime}$ \\
\hline Porto Alegre & 10,00 & $30^{\circ} 01^{\prime} 53^{\prime \prime}$ & $51^{\circ} 13^{\prime} 19^{\prime \prime}$ \\
\hline Rio Grande & 2,46 & $32^{\circ} 01^{\prime} 44^{\prime \prime}$ & $52^{\circ} 05^{\prime} 40^{\prime \prime}$ \\
\hline Santa Cruz do Sul & 52,51 & $29^{\circ} 43^{\prime} 05^{\prime \prime}$ & $52^{\circ} 25^{\prime} 45^{\prime \prime}$ \\
\hline Santa Maria & 95,00 & $29^{\circ} 41^{\prime} 25^{\prime \prime}$ & $53^{\circ} 42^{\prime} 42^{\prime \prime}$ \\
\hline Santa Rosa & 360,00 & $27^{\circ} 51^{\prime} 50^{\prime \prime}$ & $54^{\circ} 25^{\prime} 59^{\prime \prime}$ \\
\hline Santa Vitória do Palmar & 24,01 & $33^{\circ} 31^{\prime} 14^{\prime \prime}$ & $53^{\circ} 21^{\prime} 47^{\prime \prime}$ \\
\hline Santana do Livramento & 328,00 & $30^{\circ} 50^{\prime} 18^{\prime \prime}$ & $55^{\circ} 36^{\prime} 56^{\prime \prime}$ \\
\hline Santiago & 439,00 & $29^{\circ} 11^{\prime} 00^{\prime \prime}$ & $54^{\circ} 53^{\prime} 10^{\prime \prime}$ \\
\hline Santo Ângelo & 284,50 & $28^{\circ} 18^{\prime} 14^{\prime \prime}$ & $54^{\circ} 15^{\prime} 52^{\prime \prime}$ \\
\hline São Borja & 96,00 & $28^{\circ} 39^{\prime} 44^{\prime \prime}$ & $56^{\circ} 00^{\prime} 15^{\prime \prime}$ \\
\hline São Francisco de Paula & 922,00 & $29^{\circ} 20^{\prime} 00^{\prime \prime}$ & $50^{\circ} 31^{\prime} 21^{\prime \prime}$ \\
\hline São Gabriel & 124,00 & $30^{\circ} 20^{\prime} 27^{\prime \prime}$ & $54^{\circ} 19^{\prime} 01^{\prime \prime}$ \\
\hline São Luiz Gonzaga & 245,11 & $28^{\circ} 24^{\prime} 27^{\prime \prime}$ & $55^{\circ} 01^{\prime} 18^{\prime \prime}$ \\
\hline Soledade & 720,00 & $28^{\circ} 50^{\prime} 14^{\prime \prime}$ & $52^{\circ} 31^{\prime} 00^{\prime \prime}$ \\
\hline Tapes & 5,00 & $30^{\circ} 50^{\prime} 00^{\prime \prime}$ & $51^{\circ} 35^{\prime} 00^{\prime \prime}$ \\
\hline Taquara & 35,00 & $29^{\circ} 45^{\prime} 00^{\prime \prime}$ & $50^{\circ} 45^{\prime} 00^{\prime \prime}$ \\
\hline Taquarí & 76,00 & $29^{\circ} 48^{\prime} 15^{\prime \prime}$ & $51^{\circ} 49^{\prime} 30^{\prime \prime}$ \\
\hline Torres & 4,66 & $29^{\circ} 21^{\prime} 34^{\prime \prime}$ & $49^{\circ} 43^{\prime} 39^{\prime \prime}$ \\
\hline Uruguaiana & 62,31 & $29^{\circ} 45^{\prime} 23^{\prime \prime}$ & $57^{\circ} 05^{\prime} 12^{\prime \prime}$ \\
\hline Vacaria & 954,60 & $28^{\circ} 33^{\prime} 00^{\prime \prime}$ & $50^{\circ} 42^{\prime} 21^{\prime \prime}$ \\
\hline Viamão & 48,77 & $30^{\circ} 05^{\prime} 00^{\prime \prime}$ & $50^{\circ} 47^{\prime} 00^{\prime \prime}$ \\
\hline
\end{tabular}

${ }^{(1)}$ Fonte: Instituto de Pesquisas Agronômicas (1989).
Em cada município, a partir dos dados diários de temperatura mínima, medida em abrigo meteorológico situado a 1,5 m de altura em relação à superfície do solo, foi calculada a temperatura mínima média decendial e a média da temperatura mínima média decendial (Tm) do período de 1945-1974, totalizando 30 anos de observações, nos 36 decêndios do ano (1o decêndio de janeiro até 3o decêndio de dezembro).

Os dados de Tm e as coordenadas geográficas altitude, latitude e longitude, dos 41 municípios, formaram 36 matrizes (36 decêndios) de $4 \times 41$ (4 variáveis e 41 observações). Em cada uma das matrizes, realizou-se a análise de trilha (path analysis) das variáveis causais (altitude, latitude e longitude) sobre o efeito Tm. Na análise de trilha, inicialmente, estimou-se a matriz de coeficientes de correlação linear de Pearson entre as variáveis Tm, altitude, latitude e longitude, e realizou-se o diagnóstico multicolinearidade nessa matriz, visando a manter o controle sobre quais variáveis (muito correlacionados com outras) poderiam ser eliminadas do estudo para evitar estimativas de efeitos diretos e indiretos viesados na análise de trilha.

Em cada decêndio, foi usado o modelo de regressão linear múltipla com k variáveis independentes: $Y_{j}=\beta_{0}+\sum_{i=1}^{k} \beta_{i} X_{i j}+\varepsilon_{j}$, em que $Y_{j}$ é o valor observado da Tm; $\beta_{0}$ é o coeficiente linear; $\beta_{\mathrm{i}}$ é o coeficiente de regressão das variáveis independentes $X_{\mathrm{i}} ; X_{\mathrm{ij}}$ são as variáveis independentes $X_{i}$ na observação j; e $\varepsilon$ é o erro associado à variável $Y$ na observação j. Neste modelo, a variável Y é a função linear das variáveis independentes; os valores das variáveis independentes são fixos; e os erros têm média zero, são homocedásticos, independentes e de distribuição normal (Draper \& Smith, 1996; Neter et al., 1997; Hoffmann \& Vieira, 1998; Souza, 1998).

Os parâmetros do modelo das equações de regressão linear múltipla foram estimados pelo método passo a passo com teste para saída de variáveis (stepwise backward), tendo a Tm como variável dependente e as demais (altitude, latitude e longitude) como independentes. Em cada decêndio, verificou-se o ajuste dos modelos de estimativa da Tm, pelo coeficiente de correlação linear de Pearson entre a Tm estimada pelo modelo e a Tm observada. Para isso, foram usados dados de dez municípios (Encruzilhada do Sul, Júlio de Castilhos, Rio Grande, Santa Maria, Santa Rosa, São 
Borja, São Gabriel, Taquari, Vacaria e Veranópolis) do Estado do Rio Grande do Sul, de 1975 a 2004, com alguns municípios diferentes dos utilizados na obtenção das estimativas dos parâmetros do modelo. As análises foram realizadas com auxílio do programa Genes (Cruz, 2001) e do aplicativo MSOffice Excel.

\section{Resultados e Discussão}

A magnitude e o sinal do coeficiente de correlação linear de Pearson, da Tm com as variáveis altitude, latitude e longitude, foram diferentes (Tabela 2). Em todos os decêndios, houve correlação, negativa e

Tabela 2. Estimativa dos coeficientes de correlação linear de Pearson, da temperatura mínima média decendial do ar (Tm) com a altitude, a latitude e a longitude, no Estado do Rio Grande do Sul, com dados de 1945 a 1974.

\begin{tabular}{|c|c|c|c|c|}
\hline Mês & Decêndio & Altitude & Latitude & Longitude \\
\hline \multirow[t]{3}{*}{ Janeiro } & 1 & $-0,7733^{* *}$ & $0,0965^{\text {ns }}$ & $0,4382^{* * *}$ \\
\hline & 2 & $-0,8456^{* *}$ & $0,1547^{\mathrm{ns}}$ & $0,3500^{*}$ \\
\hline & 3 & $-0,8254^{* *}$ & $0,1470^{\mathrm{ns}}$ & $0,3061^{\mathrm{ns}}$ \\
\hline \multirow[t]{3}{*}{ Fevereiro } & 1 & $-0,7761^{* *}$ & $0,1069^{\text {ns }}$ & $0,3034^{\mathrm{ns}}$ \\
\hline & 2 & $-0,8139^{* *}$ & $0,1389^{\text {ns }}$ & $0,2781^{\mathrm{ns}}$ \\
\hline & 3 & $-0,8270^{* *}$ & $0,1457^{\mathrm{ns}}$ & $0,3118^{*}$ \\
\hline \multirow[t]{3}{*}{ Março } & 1 & $-0,7876^{* *}$ & $0,1327^{\mathrm{ns}}$ & $0,2615^{\mathrm{ns}}$ \\
\hline & 2 & $-0,8312^{* *}$ & $0,2260^{\mathrm{ns}}$ & $0,1695^{\mathrm{ns}}$ \\
\hline & 3 & $-0,7799^{* *}$ & $0,1415^{\mathrm{ns}}$ & $0,1323^{\mathrm{ns}}$ \\
\hline \multirow[t]{3}{*}{ Abril } & 1 & $-0,7068^{k *}$ & $0,0899^{\mathrm{ns}}$ & $0,1747^{\text {ns }}$ \\
\hline & 2 & $-0.7177^{* *}$ & $0,1056^{\mathrm{ns}}$ & $0,1462^{\mathrm{ns}}$ \\
\hline & 3 & $-0,7037^{* *}$ & $0,1840^{\text {ns }}$ & $0,1199^{\mathrm{ns}}$ \\
\hline \multirow[t]{3}{*}{ Maio } & 1 & $-0,6437^{* *}$ & $0,0908^{\mathrm{ns}}$ & $0,1315^{\mathrm{ns}}$ \\
\hline & 2 & $-0,7132^{* *}$ & $0,1710^{\mathrm{ns}}$ & $0,2261^{\mathrm{ns}}$ \\
\hline & 3 & $-0,6564^{* *}$ & $0,1587^{\mathrm{ns}}$ & $0,1926^{\mathrm{ns}}$ \\
\hline \multirow[t]{3}{*}{ Junho } & 1 & $-0,6105^{* *}$ & $-0,0330^{\mathrm{ns}}$ & $0,1100^{\mathrm{ns}}$ \\
\hline & 2 & $-0,7115^{* *}$ & $0,0653^{\mathrm{ns}}$ & $0,0836^{\mathrm{ns}}$ \\
\hline & 3 & $-0,5560^{* *}$ & $-0,1108^{\mathrm{ns}}$ & $0,1387^{\mathrm{ns}}$ \\
\hline \multirow[t]{3}{*}{ Julho } & 1 & $-0,6602^{* *}$ & $0,0528^{\mathrm{ns}}$ & $0,1351^{\mathrm{ns}}$ \\
\hline & 2 & $-0,6826^{* *}$ & $0,1285^{\text {ns }}$ & $0,2334^{\mathrm{ns}}$ \\
\hline & 3 & $-0,5785^{* *}$ & $0,0021^{\mathrm{ns}}$ & $0,1422^{\text {ns }}$ \\
\hline \multirow[t]{3}{*}{ Agosto } & 1 & $-0,6425^{* *}$ & $-0,0178^{\mathrm{ns}}$ & $0,0968^{\text {ns }}$ \\
\hline & 2 & $-0,5687^{* *}$ & $-0,0907^{\mathrm{ns}}$ & $0,3648^{*}$ \\
\hline & 3 & $-0,6155^{* *}$ & $-0,1489^{\mathrm{ns}}$ & $0,2203^{\mathrm{ns}}$ \\
\hline \multirow[t]{3}{*}{ Setembro } & 1 & $-0,5356^{* *}$ & $-0,2081^{\mathrm{ns}}$ & $0,1780^{\mathrm{ns}}$ \\
\hline & 2 & $-0,6438^{* *}$ & $-0,1532^{\mathrm{ns}}$ & $0,1348^{\mathrm{ns}}$ \\
\hline & 3 & $-0,7410^{* *}$ & $0,0459^{\mathrm{ns}}$ & $0,1846^{\mathrm{ns}}$ \\
\hline \multirow[t]{3}{*}{ Outubro } & 1 & $-0,6921^{* *}$ & $-0,0850^{\mathrm{ns}}$ & $0,1746^{\mathrm{ns}}$ \\
\hline & 2 & $-0,7208^{* *}$ & $-0,0207^{\mathrm{ns}}$ & $0,2540^{\mathrm{ns}}$ \\
\hline & 3 & $-0,7683^{* *}$ & $0,0191^{\mathrm{ns}}$ & $0,2676^{\mathrm{ns}}$ \\
\hline \multirow[t]{3}{*}{ Novembro } & 1 & $-0,7723^{* *}$ & $0,0479^{\mathrm{ns}}$ & $0,2729^{\mathrm{ns}}$ \\
\hline & 2 & $-0,7630^{* *}$ & $0,0303^{\mathrm{ns}}$ & $0,2782^{\mathrm{ns}}$ \\
\hline & 3 & $-0,7957^{* *}$ & $0,0808^{\mathrm{ns}}$ & $0,3699^{*}$ \\
\hline \multirow[t]{3}{*}{ Dezembro } & 1 & $-0,7890^{k *}$ & $0,0504^{\mathrm{ns}}$ & $0,3407^{*}$ \\
\hline & 2 & $-0,7797^{* *}$ & $0,0598^{\text {ns }}$ & $0,3204^{*}$ \\
\hline & 3 & $-0,7436^{* *}$ & $0,1104^{\mathrm{ns}}$ & $0,3806^{*}$ \\
\hline
\end{tabular}

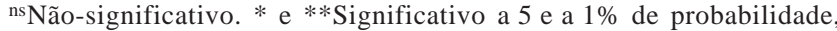
respectivamente, pelo teste t, com 39 graus de liberdade. significativa entre Tm e altitude, evidenciando diminuição da Tm com o aumento da altitude. O coeficiente de correlação entre Tm e latitude foi positivo ou negativo, de baixa magnitude e não-significativo, evidenciando não haver relação linear entre essas variáveis.

Entre Tm e longitude, houve correlação em oito decêndios e nos demais não houve significância da associação linear. Nesses últimos, com exceções, o coeficiente foi superior ao obtido entre Tm e latitude. Diante desses resultados, infere-se que num modelo de regressão linear múltipla da Tm em função da altitude, latitude e longitude, as variáveis mais correlacionadas linearmente devam estar representadas. Assim, a seguinte ordem decrescente de explicação da variação da Tm, é esperada: altitude, longitude e latitude.

A correlação é uma medida de associação entre os caracteres e avalia o grau de relacionamento linear entre variáveis. Portanto, não é possível concluir sobre as relações de causa e efeito, ou melhor, que de fato maiores altitudes e menores longitudes, nos decêndios com correlação significativa, diminuam a Tm, e ainda que a latitude não interfira na variação da Tm; ou seja, apenas por meio dos coeficientes de correlação, não é possível inferir qual das variáveis tem efeito direto na Tm. Assim, o método de análise de trilha investiga essas relações e fornece quantidades, chamadas coeficientes de trilha, que medem a influência de uma variável sobre a outra, independentemente das demais, permitindo desdobrar os coeficientes de correlações simples em efeitos diretos e indiretos (Vencovsky \& Barriga, 1992).

O diagnóstico de multicolinearidade (Cruz, 2001) entre as variáveis mostrou colinearidade fraca (Cruz \& Carneiro, 2003) nas 36 matrizes de coeficientes de correlação, o que permitiu a realização da análise de trilha sem colinearidade, não havendo necessidade de descarte de variáveis para realização dessa análise. O desdobramento da correlação total de cada uma das variáveis (altitude, latitude e longitude) sobre a variável Tm, em efeitos diretos e indiretos, esclarece a forma pela qual esses caracteres causais influem na variação da Tm (Tabela 3).

Nos 36 decêndios do ano, a variável causal altitude apresentou coeficiente de correlação linear de Pearson significativo e semelhante ao efeito direto, em magnitude e sinal, explicando a associação entre esse fator causal e o efeito Tm. Os efeitos indiretos via latitude e longitude foram, respectivamente, baixo e muito baixo. Assim, pode-se inferir que com o aumento da altitude a Tm diminui. 
Tabela 3. Estimativa dos coeficientes de correlação de Pearson e respectivas estimativas dos efeitos diretos e indiretos das variáveis altitude, latitude e longitude sobre a temperatura mínima média decendial do ar (Tm), em cada decêndio, no Estado do Rio Grande do Sul, com dados de 1945 a 1974.

\begin{tabular}{|c|c|c|c|c|c|c|c|c|c|}
\hline \multirow[t]{2}{*}{ Efeito } & Altitude & Latitude & ongitude & Altitude & Latitude & Longitude & Altitude & Latitude & Longitude \\
\hline & \multicolumn{3}{|c|}{-- Janeiro - $1^{\underline{Q}}$ decêndio --- } & \multicolumn{3}{|c|}{---- Janeiro $-2^{\mathbb{V}}$ decêndio ---- } & \multicolumn{3}{|c|}{--- Janeiro $-3^{0}$ decêndio ---- } \\
\hline Direto sobre Tm & $-0,915$ & $-0,378$ & 0.184 & -1.017 & -0.369 & 0.066 & -1.010 & $-0,373$ & 0.024 \\
\hline Indireto via altitude & & 0,470 & 0,262 & & 0,523 & 0,292 & & 0,520 & 0,290 \\
\hline Indireto via latitude & 0,194 & & $-0,008$ & 0,190 & & $-0,008$ & 0,192 & & $-0,008$ \\
\hline Indireto via longitude & $-0,053$ & 0,004 & & $-0,019$ & 0,001 & & $-0,007$ & 0,001 & \\
\hline Total (corr. Pearson) & $-0,773$ & 0,097 & 0,438 & $-0,846$ & 0,155 & 0,350 & $-0,825$ & 0,147 & 0,306 \\
\hline Coeficiente de determinação & & 0,752 & & & 0,826 & & & 0,787 & \\
\hline \multirow[t]{2}{*}{ Efeito da variável residual } & & 0,498 & & & 0,418 & & & 0,462 & \\
\hline & \multicolumn{3}{|c|}{-- Fevereiro - $1^{\varrho}$ decêndio -- } & \multicolumn{3}{|c|}{--- Fevereiro $-2^{0}$ decêndio --- } & \multicolumn{3}{|c|}{---- Fevereiro $-3^{0}$ decêndio ---- } \\
\hline Direto sobre Tm & $-0,968$ & $-0,391$ & 0,034 & $-1,011$ & $-0,381$ & $-0,004$ & $-1,011$ & $-0,375$ & 0,030 \\
\hline Indireto via altitude & & 0,498 & 0,277 & & 0,520 & 0,290 & & 0,520 & 0,290 \\
\hline Indireto via latitude & 0,201 & & $-0,008$ & 0,196 & & $-0,008$ & 0,193 & & $-0,008$ \\
\hline Indireto via longitude & $-0,010$ & 0,001 & & 0,001 & 0,000 & & $-0,009$ & 0,001 & \\
\hline Total (corr. Pearson) & $-0,776$ & 0,107 & 0,303 & $-0,814$ & 0,139 & 0,278 & $-0,827$ & 0,146 & 0,312 \\
\hline Coeficiente de determinação & & 0,719 & & & 0,769 & & & 0,791 & \\
\hline \multirow{2}{*}{ Efeito da variável residual } & & 0,530 & & & 0,481 & & & 0,457 & \\
\hline & \multicolumn{3}{|c|}{---- Março - $1^{0}$ decêndio ---- } & \multicolumn{3}{|c|}{----- Março - $2^{0}$ decêndio ----- } & \multicolumn{3}{|c|}{---- Março - $3^{0}$ decêndio ----- } \\
\hline Direto sobre Tm & $-0,983$ & $-0,372$ & $-0,013$ & $-1,015$ & $-0,294$ & $-0,116$ & $-1,018$ & $-0,379$ & $-0,152$ \\
\hline Indireto via altitude & & 0,505 & 0,282 & & 0,522 & 0,291 & & 0,524 & 0,292 \\
\hline Indireto via latitude & 0,191 & & $-0,008$ & 0,151 & & $-0,006$ & 0,195 & & $-0,008$ \\
\hline Indireto via longitude & 0,004 & 0,000 & & 0,033 & $-0,002$ & & 0,044 & $-0,003$ & \\
\hline Total (corr. Pearson) & $-0,788$ & 0,133 & 0,262 & $-0,831$ & 0,226 & 0,170 & $-0,780$ & 0,142 & 0,132 \\
\hline Coeficiente de determinação & & 0,721 & & & 0,758 & & & 0,720 & \\
\hline \multirow[t]{2}{*}{ Efeito da variável residual } & & 0,528 & & & 0,492 & & & 0,529 & \\
\hline & \multicolumn{3}{|c|}{---- Abril - $1^{-}$decêndio ---- } & \multicolumn{3}{|c|}{------ Abril - $2^{\underline{\underline{Q}}}$ decêndio ------ } & \multicolumn{3}{|c|}{------ Abril - $3^{\circ}$ decêndio ------ } \\
\hline Direto sobre $\mathrm{Tm}$ & $-0,930$ & $-0,386$ & $-0,084$ & -0.946 & $-0,378$ & -0.117 & $-0,875$ & -0.263 & $-0,126$ \\
\hline Indireto & & 0,478 & 0,267 & & 0,486 & 0,271 & & 0,450 & 0,251 \\
\hline Indireto via latitude & 0,199 & & $-0,008$ & 0,195 & & $-0,008$ & 0,135 & & $-0,005$ \\
\hline Indireto via longitude & 0,024 & $-0,002$ & & 0,034 & $-0,002$ & & 0,036 & $-0,003$ & \\
\hline Total (corr. Pearson) & $-0,707$ & 0,090 & 0,175 & $-0,718$ & 0,106 & 0,146 & $-0,704$ & 0,184 & 0,120 \\
\hline Coeficiente de determinação & & 0,608 & & & 0,622 & & & 0,552 & \\
\hline Efeito da variável residual & & 0,626 & & & 0,615 & & & 0,669 & \\
\hline & --- Ma & $o-1^{0} \mathrm{dec}$ & dio ---- & ------ M & $p-2^{\underline{0}} \mathrm{dec}$ & ndio ------ & ------ M & $0-3^{-0} \mathrm{dec}$ & dio------ \\
\hline Direto sobre Tm & $-0,851$ & $-0,345$ & $-0,106$ & $-0,855$ & $-0,268$ & $-0,014$ & $-0,793$ & $-0,248$ & $-0,030$ \\
\hline Indireto via altitude & & 0,438 & 0,244 & & 0,440 & 0,245 & & 0,408 & 0,227 \\
\hline Indireto via latitude & 0,177 & & $-0,007$ & 0,138 & & $-0,006$ & 0,128 & & $-0,005$ \\
\hline Indireto via longitude & 0,030 & $-0,002$ & & 0,004 & 0,000 & & 0,009 & $-0,001$ & \\
\hline Total (corr. Pearson) & $-0,644$ & 0,091 & 0,132 & $-0,713$ & 0,171 & 0,226 & $-0,656$ & 0,159 & 0,193 \\
\hline Coeficiente de determinação & & 0,503 & & & 0,561 & & & 0,475 & \\
\hline Efeito da variável residual & & 0,705 & & & 0,663 & & & 0,725 & \\
\hline & ---- Jun & $10-1^{\underline{g}} \mathrm{dec}$ & ndio ---- & ------ Jur & $0-2^{\circ} \mathrm{dec}$ & ndio ------ & ------ Jur & $0-3^{\frac{0}{d e c}}$ & dio -.---- \\
\hline Direto sobre Tm & $-0,905$ & $-0,496$ & $-0,140$ & $-0,994$ & $-0,442$ & $-0,192$ & $-0,871$ & $-0,557$ & $-0,100$ \\
\hline Indireto via altitude & & 0,466 & 0,260 & & 0,511 & 0,285 & & 0,448 & 0,250 \\
\hline Indireto via latitude & 0,255 & & $-0,010$ & 0,227 & & $-0,009$ & 0,286 & & $-0,011$ \\
\hline Indireto via longitude & 0,040 & $-0,003$ & & 0,055 & $-0,004$ & & 0,029 & $-0,002$ & \\
\hline Total (corr. Pearson) & $-0,611$ & $-0,033$ & 0,110 & $-0,712$ & 0,065 & 0,084 & $-0,556$ & $-0,111$ & 0,139 \\
\hline Coeficiente de determinação & & 0,554 & & & 0,662 & & & 0,532 & \\
\hline Efeito da variável residual & & 0,668 & & & 0,581 & & & 0,684 & \\
\hline & ---- Jull & $o-1^{0} \mathrm{dec}$ & adio ---- & ------ Jul & $-2^{\circ} \operatorname{dec}$ & Idio ------ & ------ Ju & $0-3^{11} \mathrm{de}$ & dio------ \\
\hline Direto & $-0,904$ & $-0,410$ & $-0,116$ & -0.839 & -0.303 & $-0,001$ & $-0,816$ & $-0,416$ & $-0,083$ \\
\hline Indireto via altitude & & 0,465 & 0,259 & & 0,431 & 0,240 & & 0,420 & 0,234 \\
\hline Indireto via latitude & 0,211 & & $-0,008$ & 0,156 & & $-0,006$ & 0,214 & & $-0,009$ \\
\hline Indireto via longitude & 0,033 & $-0,002$ & & 0,000 & 0,000 & & 0,024 & $-0,002$ & \\
\hline Total (corr. Pearson) & $-0,660$ & 0,053 & 0,135 & $-0,683$ & 0,129 & 0,233 & $-0,579$ & 0,002 & 0,142 \\
\hline Coeficiente de determinação & & 0,560 & & & 0,533 & & & 0,459 & \\
\hline Efeito da variável residual & & 0,664 & & & 0,683 & & & 0,735 & \\
\hline
\end{tabular}


Tabela 3. Continuação...

\begin{tabular}{|c|c|c|c|c|c|c|c|c|c|}
\hline \multirow[t]{2}{*}{ Efeito } & Altitude & Latitud & Longitude & Altitude & Latitude & Longitude & Altitude & Latitude & Longitude \\
\hline & \multicolumn{3}{|c|}{ - Agosto - $1^{0}$ decêndio -...-. } & \multicolumn{3}{|c|}{---- Agosto - $2^{0}$ decêndio ---- } & \multicolumn{3}{|c|}{---- Agosto $-3^{\underline{Q}}$ decêndio ----- } \\
\hline Direto sobre Tm & $-0,948$ & $-0,502$ & $-0,165$ & $-0,780$ & $-0,495$ & 0,151 & $-0,956$ & $-0,640$ & $-0,041$ \\
\hline Indireto via altitude & & 0,487 & 0,272 & & 0,401 & 0,224 & & 0,492 & 0,274 \\
\hline Indireto via latitude & 0,258 & & $-0,010$ & 0,254 & & $-0,010$ & 0,329 & & $-0,013$ \\
\hline Indireto via longitude & 0,047 & $-0,003$ & & $-0,043$ & 0,003 & & 0,012 & $-0,001$ & \\
\hline Total (corr. Pearson) & $-0,643$ & $-0,018$ & 0,097 & $-0,569$ & $-0,091$ & 0,365 & $-0,616$ & $-0,149$ & 0,220 \\
\hline Coeficiente de determinação & & 0,602 & & & 0,543 & & & 0,675 & \\
\hline \multirow{2}{*}{ Efeito da variável residual } & & 0,631 & & & 0,676 & & & 0,570 & \\
\hline & \multicolumn{3}{|c|}{---- Setembro - $1^{\underline{0}}$ decêndio ---- } & \multicolumn{3}{|c|}{----- Setembro $-2^{\underline{Q}}$ decêndio ----- } & \multicolumn{3}{|c|}{----- Setembro $-3^{\underline{0}}$ decêndio ----- } \\
\hline Direto sobre Tm & $-0,898$ & $-0,669$ & $-0,066$ & $-1,038$ & $-0,684$ & $-0,149$ & $-1,011$ & $-0,472$ & $-0,096$ \\
\hline Indireto via altitude & & 0,462 & 0,258 & & 0,534 & 0,298 & & 0,520 & 0,290 \\
\hline Indireto via latitude & 0,344 & & $-0,014$ & 0,352 & & $-0,014$ & 0,243 & & $-0,010$ \\
\hline Indireto via longitude & 0,019 & $-0,001$ & & 0,043 & $-0,003$ & & 0,027 & $-0,002$ & \\
\hline Total (corr. Pearson) & $-0,536$ & $-0,208$ & 0,178 & $-0,644$ & $-0,153$ & 0,135 & $-0,741$ & 0,046 & 0,185 \\
\hline Coeficiente de determinação & & 0,609 & & & 0,753 & & & 0,710 & \\
\hline \multirow[t]{2}{*}{ Efeito da variável residual } & & 0,626 & & & 0,497 & & & 0,539 & \\
\hline & \multicolumn{3}{|c|}{---- Outubro - $1^{11}$ decêndio ----- } & \multicolumn{3}{|c|}{----- Outubro $-2^{\underline{Q}}$ decêndio ----- } & \multicolumn{3}{|c|}{----- Outubro $-3^{0}$ decêndio ------ } \\
\hline Direto sobre $\mathrm{Tm}$ & $-1,042$ & $-0,619$ & $-0,112$ & $-1,003$ & $-0,536$ & $-0,023$ & $-1,038$ & $-0,515$ & $-0,020$ \\
\hline Indir & & 0,536 & 0,299 & & 0,516 & 0,288 & & 0,534 & 0,298 \\
\hline Indiret & 0,318 & & $-0,013$ & 0,276 & & $-0,011$ & 0,265 & & $-0,011$ \\
\hline Indireto via longitude & 0,032 & $-0,002$ & & 0,007 & $-0,001$ & & 0,006 & 0,000 & \\
\hline Total (corr. Pearson) & $-0,692$ & $-0,085$ & 0,175 & $-0,721$ & $-0,021$ & 0,254 & $-0,768$ & 0,019 & 0,268 \\
\hline Coeficiente de determinação & & 0,754 & & & 0,728 & & & 0,783 & \\
\hline \multirow[t]{2}{*}{ Efeito da variável residual } & & 0,496 & & & 0,521 & & & 0,466 & \\
\hline & \multicolumn{3}{|c|}{--- Novembro - $1^{\circ}$ decêndio --- } & \multicolumn{3}{|c|}{---- Novembro $-2^{\underline{0}}$ decêndio ---- } & \multicolumn{3}{|c|}{--- Novembro $-3^{\circ}$ decêndio ----- } \\
\hline Direto sobre Tm & $-1,020$ & $-0,477$ & $-0,010$ & $-1,017$ & $-0,493$ & $-0,004$ & $-0,990$ & $-0,430$ & 0,095 \\
\hline Indireto via altitude & & 0,525 & 0,293 & & 0,523 & 0,292 & & 0,509 & 0,284 \\
\hline Indireto via latitude & 0,245 & & $-0,010$ & 0,253 & & $-0,010$ & 0,221 & & $-0,009$ \\
\hline Indireto via longitude & 0,003 & 0,000 & & 0,001 & 0,000 & & $-0,027$ & 0,002 & \\
\hline Total (corr. Pearson) & $-0,772$ & 0,048 & 0,273 & $-0,763$ & 0,030 & 0,278 & $-0,796$ & 0,081 & 0,370 \\
\hline Coeficiente de determinação & & 0,762 & & & 0,760 & & & 0,788 & \\
\hline \multirow[t]{2}{*}{ Efeito da variável residual } & & 0,487 & & & 0,490 & & & 0,461 & \\
\hline & \multicolumn{3}{|c|}{--- Dezembro - $1^{0}$ decêndio ---- } & \multicolumn{3}{|c|}{---- Dezembro $-2^{\underline{O}}$ decêndio ---- } & \multicolumn{3}{|c|}{---- Dezembro $-3^{\underline{\underline{n}}}$ decêndio ----- } \\
\hline Direto so & $-1,015$ & $-0,473$ & 0,059 & $-1,002$ & $-0,456$ & 0,042 & $-0,883$ & $-0,346$ & 0,134 \\
\hline Indireto via altitude & & 0,522 & 0,291 & & 0,515 & 0,287 & & 0,454 & 0,253 \\
\hline Indireto via latitude & 0,243 & & $-0,010$ & 0,235 & & $-0,009$ & 0,178 & & $-0,007$ \\
\hline Indireto via longitude & $-0,017$ & 0,001 & & $-0,012$ & 0,001 & & $-0,039$ & 0,003 & \\
\hline Total (corr. Pearson) & $-0,789$ & 0,050 & 0,341 & $-0,780$ & 0,060 & 0,320 & $-0,744$ & 0,110 & 0,381 \\
\hline Coeficiente de determinação & & 0,797 & & & 0,768 & & & 0,670 & \\
\hline Efeito da variável residual & & 0,450 & & & 0,482 & & & 0,575 & \\
\hline
\end{tabular}

A variável causal latitude, cujo coeficiente de correlação linear de Pearson foi não-significativo e de baixa magnitude, apresentou efeito direto negativo e alto, sobre a Tm, nos 36 decêndios, evidenciando diminuição da Tm com o aumento da latitude. O efeito indireto positivo e de alta magnitude via altitude, sobre a Tm, impediu a manifestação dessa correlação. Portanto, pode-se inferir que, no Rio Grande do Sul, existe diminuição da Tm com o aumento da latitude, isto é, essa diminuição ocorre no sentido norte-sul do Estado.

Quanto à variável causal longitude, que apresentou coeficiente de correlação linear de Pearson positivo e com magnitude - em alguns casos, significativa a $1 \%$-, o efeito direto sobre a Tm foi negligível na maioria dos decêndios, evidenciando quase inexistência de associação linear entre essas variáveis. O efeito indireto positivo e de alta magnitude via altitude sobre a Tm impediu a manifestação dessa correlação. Pode-se inferir que, no Rio Grande do Sul, a longitude não influencia a variação da Tm. Diante disso, espera-se que no modelo de estimativa da Tm, a altitude e a latitude estejam presentes e, em decêndios com efeito direto de alta magnitude da longitude sobre a Tm, esta também conste no modelo.

A estimativa da equação de regressão linear múltipla da Tm, com exceção do primeiro decêndio de janeiro, segundo de junho e segundo de setembro, foi calculada em função da altitude e latitude, sem contribuição da longitude para melhoria da estimativa da Tm (Tabela 4). 
A variável latitude, apesar de não estar correlacionada com a Tm (Tabela 2), entrou na equação de cada decêndio, provavelmente em virtude de seu efeito direto sobre a Tm (Tabela 3). Por sua vez, a longitude, com coeficiente de correlação linear positivo e alguns decêndios significativos com a Tm, participou da equação em somente três casos, o que pode ser explicado pela presença de efeito direto baixo e efeito indireto via altitude alto. Isto comprova que a altitude e latitude são as variáveis que influenciaram na Tm de forma proporcional em todos os decêndios. Desse modo, no Rio Grande do Sul, locais com maior altitude, localizados mais ao norte do Estado, têm Tm menor; a Tm aumenta no sentido norte-sul e com o decréscimo da altitude. Há diminuição de aproximadamente 0,3 a $0,5^{\circ} \mathrm{C}$ a cada acréscimo de $100 \mathrm{~m}$ de altitude, e de 0,2 a $0,6^{\circ} \mathrm{C}$ a cada grau de aumento na latitude. Resultados semelhantes foram obtidos por Alfonsi et al. (1974).

Os modelos estimam reduções menores nas estações de outono e inverno e maiores nas estações de primavera e verão. Esse resultado pode ser explicado pela menor e maior amplitude de variação da temperatura, respectivamente, nas estações de outono, inverno e primavera, e verão. $\mathrm{O}$ coeficiente de determinação $\left(\mathrm{R}^{2}\right)$ representa a porcentagem da variação da Tm explicada pelas variáveis que constam no modelo. Assim, nas

Tabela 4. Estimativa dos parâmetros $\left(\beta_{0}, \beta_{1}, \beta_{2}\right.$ e $\left.\beta_{3}\right)$ da regressão linear múltipla ${ }^{(1)}$ e coeficiente de determinação $\left(R^{2}\right)$, da temperatura mínima média decendial do ar (Tm) em função da altitude (m), latitude (graus) e longitude (graus), no Estado do Rio Grande do Sul, com dados de 1945 a 1974.

\begin{tabular}{|c|c|c|c|c|c|c|}
\hline Mês & Decêndio & Constante $\left(\beta_{0}\right)$ & Altitude $\left(\beta_{1}\right)$ & Latitude $\left(\beta_{2}\right)$ & Longitude $\left(\beta_{3}\right)$ & $\mathrm{R}^{2}(\%)$ \\
\hline \multirow{3}{*}{ Janeiro } & 1 & 23,49115552 & $-0,00467690$ & $-0,42233972$ & 0,15916284 & 75 \\
\hline & 2 & 32,40570652 & $-0,00545713$ & $-0,43646904$ & & 82 \\
\hline & 3 & 32,80464739 & $-0,00532743$ & $-0,43120421$ & & 79 \\
\hline \multirow[t]{3}{*}{ Fevereiro } & 1 & 33,31401654 & $-0,00512719$ & $-0,45438281$ & & 72 \\
\hline & 2 & 31,94391742 & $-0,00494153$ & $-0,40702872$ & & 77 \\
\hline & 3 & 31,94686841 & $-0,00514537$ & $-0,41832663$ & & 79 \\
\hline \multirow[t]{3}{*}{ Março } & 1 & 30,91485678 & $-0,00479367$ & $-0,39690054$ & & 72 \\
\hline & 2 & 26,52174058 & $-0,00469331$ & $-0,28913871$ & & 75 \\
\hline & 3 & 28,66759839 & $-0,00467010$ & $-0,37489731$ & & 70 \\
\hline \multirow[t]{3}{*}{ Abril } & 1 & 28,82785943 & $-0,00473542$ & $-0,42891867$ & & 60 \\
\hline & 2 & 26,35700088 & $-0,00451013$ & $-0,39172744$ & & 61 \\
\hline & 3 & 21,03004708 & $-0,00403038$ & $-0,25734199$ & & 54 \\
\hline \multirow[t]{3}{*}{ Maio } & 1 & 22,24957212 & $-0,00370234$ & $-0,32581704$ & & 49 \\
\hline & 2 & 20,16644287 & $-0,00397090$ & $-0,27183798$ & & 56 \\
\hline & 3 & 19,04585803 & $-0,00370098$ & $-0,25183922$ & & 47 \\
\hline \multirow[t]{3}{*}{ Junho } & 1 & 24,29381401 & $-0,00374009$ & $-0,45230103$ & & 54 \\
\hline & 2 & 27,70044110 & $-0,00388304$ & $-0,37756744$ & $-0,12741114$ & 66 \\
\hline & 3 & 23,67914754 & $-0,00328719$ & $-0,46537418$ & & 52 \\
\hline \multirow[t]{3}{*}{ Julho } & 1 & 18,90404926 & $-0,00332274$ & $-0,32912116$ & & 55 \\
\hline & 2 & 17,26727432 & $-0,00330367$ & $-0,26078630$ & & 53 \\
\hline & 3 & 20,40227946 & $-0,00323438$ & $-0,36185748$ & & 45 \\
\hline \multirow[t]{3}{*}{ Agosto } & 1 & 22,17175390 & $-0,00370921$ & $-0,43347276$ & & 58 \\
\hline & 2 & 24,25274572 & $-0,00334717$ & $-0,45589315$ & & 52 \\
\hline & 3 & 29,29463961 & $-0,00410409$ & $-0,60368532$ & & 67 \\
\hline \multirow[t]{3}{*}{ Setembro } & 1 & 28,92631124 & $-0,00354892$ & $-0,58413035$ & & 60 \\
\hline & 2 & 38,18057022 & $-0,00455576$ & $-0,65658799$ & $-0,1107918$ & 75 \\
\hline & 3 & 26,03131487 & $-0,00418495$ & $-0,42759114$ & & 70 \\
\hline \multirow[t]{3}{*}{ Outubro } & 1 & 31,90919427 & $-0,00463348$ & $-0,60731695$ & & 74 \\
\hline & 2 & 29,43232359 & $-0,00448609$ & $-0,52499294$ & & 73 \\
\hline & 3 & 31,38007132 & $-0,00509487$ & $-0,55241939$ & & 78 \\
\hline \multirow[t]{3}{*}{ Novembro } & 1 & 31,76058213 & $-0,00537202$ & $-0,54889069$ & & 76 \\
\hline & 2 & 31,65800635 & $-0,00495700$ & $-0,52525065$ & & 76 \\
\hline & 3 & 32,66918800 & $-0,00545823$ & $-0,51977575$ & & 78 \\
\hline \multirow[t]{3}{*}{ Dezembro } & 1 & 34,82406793 & $-0,00557860$ & $-0,56819753$ & & 79 \\
\hline & 2 & 34,01012299 & $-0,00542831$ & $-0,54077382$ & & 77 \\
\hline & 3 & 30,94936694 & $-0,00490999$ & $-0,42520485$ & & 65 \\
\hline
\end{tabular}

${ }^{(1)}$ Método passo a passo com teste para saída de variáveis (stepwise backward); $\mathrm{PR}>\mathrm{F}$ para entrar e/ou para sair = 10\%. 
estações de outono e inverno, outras variáveis não integrantes do modelo exercem maior porcentagem de explicação na variação da Tm que nas estações de primavera e verão. Valores menores de coeficientes de determinação nos meses de inverno são relatados por Pinto \& Alfonsi (1974).

O coeficiente de correlação linear entre a Tm estimada e a Tm observada em dez municípios do Rio Grande do Sul, utilizado para validação dos modelos obtidos, oscilou entre 0,83 e 0,97 nos 36 decêndios do ano, com média de 0,93 entre os decêndios, comprovando a adequabilidade dos modelos (Tabela 5). A distribuição

Tabela 5. Estimativa dos coeficientes de correlação linear de Pearson, da temperatura mínima média decendial do ar (Tm) estimada em função da altitude, latitude e longitude, e a Tm observada em dez municípios do Estado do Rio Grande do Sul, com dados de 1975 a 2004.

\begin{tabular}{|c|c|c|}
\hline Mês & Decêndio & Tm estimada $\mathrm{x}$ Tm observada \\
\hline \multirow[t]{3}{*}{ Janeiro } & 1 & $0,97^{* *}$ \\
\hline & 2 & $0,96^{* *}$ \\
\hline & 3 & $0,97^{* *}$ \\
\hline \multirow[t]{3}{*}{ Fevereiro } & 1 & $0,96^{* *}$ \\
\hline & 2 & $0,96^{* *}$ \\
\hline & 3 & $0,95^{* *}$ \\
\hline \multirow[t]{3}{*}{ Março } & 1 & $0,94^{* *}$ \\
\hline & 2 & $0,93^{* *}$ \\
\hline & 3 & $0,91^{* *}$ \\
\hline \multirow[t]{3}{*}{ Abril } & 1 & $0,89^{* *}$ \\
\hline & 2 & $0,94^{* *}$ \\
\hline & 3 & $0,90^{* *}$ \\
\hline \multirow[t]{3}{*}{ Maio } & 1 & $0,91^{* *}$ \\
\hline & 2 & $0,91^{* *}$ \\
\hline & 3 & $0,89^{* *}$ \\
\hline \multirow[t]{3}{*}{ Junho } & 1 & $0,90^{* *}$ \\
\hline & 2 & $0,83^{* *}$ \\
\hline & 3 & $0,90^{* *}$ \\
\hline \multirow[t]{3}{*}{ Julho } & 1 & $0,90^{* *}$ \\
\hline & 2 & $0,88^{* *}$ \\
\hline & 3 & $0,93^{* *}$ \\
\hline \multirow[t]{3}{*}{ Agosto } & 1 & $0,93^{* *}$ \\
\hline & 2 & $0,93^{* *}$ \\
\hline & 3 & $0,90^{* *}$ \\
\hline \multirow[t]{3}{*}{ Setembro } & 1 & $0,87^{* *}$ \\
\hline & 2 & $0,90^{* *}$ \\
\hline & 3 & $0,94^{* *}$ \\
\hline \multirow[t]{3}{*}{ Outubro } & 1 & $0,93^{* *}$ \\
\hline & 2 & $0,93^{* *}$ \\
\hline & 3 & $0,96^{* *}$ \\
\hline \multirow[t]{3}{*}{ Novembro } & 1 & $0,94^{* *}$ \\
\hline & 2 & $0,95^{* *}$ \\
\hline & 3 & $0,95^{* *}$ \\
\hline \multirow[t]{3}{*}{ Dezembro } & 1 & $0,96^{* *}$ \\
\hline & 2 & $0,95^{* *}$ \\
\hline & 3 & $0,96^{* *}$ \\
\hline
\end{tabular}

**Significativo a $1 \%$ de probabilidade pelo teste $\mathrm{t}$, com 8 graus de liberdade. espacial dos 41 municípios cujos dados foram utilizados para gerar os modelos de estimativa de Tm, representou a amplitude de variação da altitude, latitude e longitude do Estado do Rio Grande do Sul. Portanto, esses modelos podem ser utilizados para estimar a Tm de qualquer ponto do Estado do Rio Grande do Sul em função das coordenadas geográficas, em todos os decêndios do ano.

Assim, em determinado local que tenha altitude de $50 \mathrm{~m}$, latitude $30^{\circ} 10^{\prime} 30^{\prime \prime} \mathrm{S}$ e longitude $50^{\circ} 48^{\prime} 10^{\prime \prime} \mathrm{W}$, a estimativa da Tm para o primeiro decêndio de janeiro é $18,6^{\circ} \mathrm{C}$ e é obtida pela equação linear múltipla (Tabela 4), conforme o exemplo:

$\mathrm{Tm}=\beta_{0}+\beta_{1}$. altitude $(\mathrm{m})+\beta_{2}$.latitude (graus) $+\beta_{3}$.longitude (graus), ou seja, Tm $=23,49115552-$ $0,0046769.50-0,42233972 \cdot 30,175000+$ $0,15916284.50,802778=18,6^{\circ} \mathrm{C}$.

\section{Conclusões}

1. A temperatura mínima média decendial do ar, no Estado do Rio Grande do Sul, em qualquer local e decêndio, pode ser estimada por meio das coordenadas geográficas.

2. A altitude e a latitude, nessa ordem, exercem maior efeito sobre a temperatura mínima média decendial do ar no Estado do Rio Grande do Sul.

\section{Referências}

ALFONSI, R.R.; PINTO, H.S.; PEDRO JÚNIOR, M.J. Estimativas das normais de temperaturas média mensal e anual do Estado de Goiás (BR) em função de altitude e latitude. Caderno de Ciências da Terra, v.45, p.1-6, 1974.

BURIOL, G.A.; ESTEFANEL, V.; FERREIRA, M.; PINTO, H.S. Estimativa das médias das temperaturas máximas mensais e anuais do Estado do Rio Grande do Sul. Revista do Centro de Ciências Rurais, v.3, p.131-150, 1973.

BURIOL, G.A.; FERREIRA, M.; ESTEFANEL, V.; PIGNATARO, I.A.B. Estimativa das médias das temperaturas máximas mensais e anuais do Estado de Santa Catarina. Revista do Centro de Ciências Rurais, v.4, p.81-102, 1974.

COELHO, D.T.; SEDIYAMA, G.C.; VIEIRA, M. Estimativa das temperaturas médias mensais e anual no Estado de Minas Gerais. Revista Ceres, v.20, p.455-459, 1973.

CRUZ, C.D. Programa GENES: versão Windows; aplicativo computacional em genética e estatística. Viçosa: UFV, 2001. 648p.

CRUZ, C.D.; CARNEIRO, P.C.S. Modelos biométricos aplicados ao melhoramento genético. Viçosa: UFV, 2003. 2v.

DRAPER, N.R.; SMITH, R. Applied regression analysis. $3^{\text {rd }}$ ed. New York: Wiley, 1996. 706p. 
ESTEFANEL, V.; FERREIRA, M.; BURIOL, G.A.; PINTO, H.S. Estimativa das médias das temperaturas mínimas mensais e anuais do Estado do Rio Grande do Sul. Revista do Centro de Ciências Rurais, v.3, p.1-20, 1973.

FEITOZA, L.R.; SCÁRDUA, J.A.; SEDIYAMA, G.C.; VALLE, S.S. Estimativas das temperaturas médias das máximas mensais e anual do Estado do Espírito Santo. Revista do Centro de Ciências Rurais, v.10, p.25-32, 1980b.

FEITOZA, L.R.; SCÁRDUA, J.A.; SEDIYAMA, G.C.; VALLE, S.S. Estimativas das temperaturas médias das mínimas mensais e anual do Estado do Espírito Santo. Revista do Centro de Ciências Rurais, v.10, p.15-24, 1980a.

FERREIRA, M.; BURIOL, G.A.; ESTEFANEL, V.; PINTO, H.S. Estimativa das temperaturas médias mensais e anuais do Estado do Rio Grande do Sul. Revista do Centro de Ciências Rurais, v.1, p.21-52, 1971.

FERREIRA, M.; BURIOL, G.A.; PIGNATARO, I.A.B.; ESTEFANEL, V. Estimativa das temperaturas médias mensais e anuais do Estado de Santa Catarina. Revista do Centro de Ciências Rurais, v.4, p.19-38, 1974.

HOFFMANN, R.; VIEIRA, S. Análise de regressão: uma introdução à econometria. 3.ed. São Paulo: Hucitec/Edusp, 1998. 379p.

INSTITUTO DE PESQUISAS AGRONÔMICAS. Secção de Ecologia Agrícola. Atlas agroclimático do Estado do Rio Grande do Sul. Porto Alegre, 1989. 3v.

LIMA, M.G.; RIBEIRO, V.Q. Equações de estimativa da temperatura do ar para o Estado do Piauí. Revista Brasileira de Agrometeorologia, v.6, p.221-227, 1998.

MEDEIROS, S.S.; CECILIO, R.A.; MELO JÚNIOR, J.C.F.; SILVA JUNIOR, J.L.C. Estimativa e espacialização das temperaturas do ar mínimas, médias e máximas na Região Nordeste do Brasil. Revista Brasileira de Engenharia Agrícola e Ambiental, v.9, p.247-255, 2005.
NETER, J.; KUTNER, M.H.; NACHTSHEIM, C.J.; WASSERMAN, W. Applied linear regression models. $3^{\text {rd }}$ ed. New York: McGraw Hill, 1997. 532p.

OLIVEIRA NETO, S.N. de; REIS, G.G. dos; REIS, M. das G.F.; LEITE, H.G.; COSTA, J.M.N. da. Estimativa de temperaturas mínima, média e máxima do território brasileiro situado entre 16 e $24^{\circ}$ latitude sul e 48 e $60^{\circ}$ longitude oeste. Engenharia na Agricultura, v.10, p.8-17, 2002.

PEDRO JÚNIOR, M.J.; MELLO, M.H.D.A.; ORTOLANI, A.A.; ALFONSI, R.R.; SENTELHAS, P.C. Estimativa das temperaturas médias mensais das máximas e das mínimas para o Estado de São Paulo. Campinas: Instituto Agronômico, 1991. 11p. (Boletim técnico, 142).

PINTO, H.S.; ALFONSI, R.R. Estimativa das temperaturas médias, máximas e mínimas mensais no Estado do Paraná, em função de altitude e latitude. Caderno de Ciências da Terra, v.52, p.1-28, 1974.

PINTO, H.S.; ORTOLANI, A.A.; ALFONSI, R.R. Estimativa das temperaturas médias mensais do Estado de São Paulo em função de altitude e latitude. Caderno de Ciências da Terra, v.23, p.1-20, 1972.

SEDIYAMA, G.C.; MELO JÚNIOR, J.C.F. Modelos para estimativa das temperaturas normais mensais médias, máximas, mínimas e anual no Estado de Minas Gerais. Engenharia na Agricultura, v.6, p.57-61, 1998.

SOUZA, G.S. Introdução aos modelos de regressão linear e não-linear. Brasília: Embrapa-SPI/Embrapa-SEA, 1998. 505p.

SPIEGEL, R.A.; SCHILLER, J.; SRINIVASAN, R.A. Probabilidade e estatística. 2.ed. Porto Alegre: Bookman, 2004. 398p.

STEEL, R.G.D.; TORRIE, J.H.; DICKEY, D.A. Principles and procedures of statistics. $3^{\text {rd }}$ ed. Nova York: McGraw Hill Book, 1997. 666p.

VENCOVSKY, R.; BARRIGA, P. Genética biométrica no fitomelhoramento. Ribeirão Preto: Sociedade Brasileira de Genética, 1992. 496p.

Recebido em 3 de março de 2005 e aprovado em 25 de janeiro de 2006 